\title{
Doxycycline and Ribavirin as Antiviral and Novel Combinational Drug to Treat Dengue, Chikungunya and as the Most Potent Antivirals for COVID-19
}

\section{Rajarajan Swaminathan ${ }^{1 *}$, Shanthi Sabarimurugan ${ }^{2}$, Sangeetha Kothandam $^{3}$ and Indu Purushothaman ${ }^{4}$}

${ }^{1}$ Professor, PG and Research, Department of Microbiology and Biotechnology

Presidency College (Aut), University of Madras and Centre for Drug Design, Discovery

and Development of Drug, SRM University, Haryana, New Delhi, India

${ }^{2}$ School of Biomedical Sciences, Faculty of Health and Biomedical Sciences, QE II

Medical Centre Campus, The University of Western Australia, Perth, Australia

${ }^{3}$ Associate Lecturer, Bharath Institute of Higher Education and Research, Chennai,

India

${ }^{4} \mathrm{PG}$ and Research, Department of Microbiology and Biotechnology, Presidency College

(Aut), University of Madras, India

*Corresponding Author: Rajarajan Swaminathan, Professor, PG and Research,

Department of Microbiology and Biotechnology Presidency College (Aut), University

of Madras and Centre for Drug Design, Discovery and Development of Drug, SRM

University, Haryana, New Delhi, India.
Received: December 17, 2021

Published: January 27, 2022

(C) All rights are reserved by Rajarajan

Swaminathan., et al.

\begin{abstract}
Objectives: To discover an effective antiviral for the treatment of Dengue and Chikungunya by in-silico and in-vitro antiviral studies. To detect a highly potent antiviral by in-silico evaluation to combat the unabated prevalence of COVID-19 as the second wave despite ongoing vaccination for emergency use.

Methods: The in-silico antiviral studies on Dengue and CHIKV have been studied through molecular docking and confirmed through in-vitro studies. The minimum nontoxic concentration of Doxycycline and Ribavirin were evaluated by cytotoxicity assay and the efficacy of monotherapy and combinational therapy was experimentally evaluated against CHIKV and Dengue serotypes in Vero cell lines. The statistical analysis was done through GraphPad Prism. Along with Doxycycline and Ribavirin, another five drugs have been studied for the anti-COVID-19 effect through virtual screening. The 3D structure of the SARS-CoV-2 main protease protein was retrieved from PDB and docked with compounds using Auto Dock 4.2.

Results: In-silico docking efficacy of Doxycycline and Ribavirin were justified by the results of in-vitro antiviral assay as monotherapy on Dengue and CHIKV. Interestingly the combinational efficacy of Doxycycline and Ribavirin was slightly superior to the monotherapy MIC value. Interestingly the combinational effect of Doxycycline and Ribavirin exhibited superior synergetic inhibitory activity than individual drug efficacy. The combinational antiviral efficacy as MIC against CHIKV strains were 62.5 and $125 \mu \mathrm{g} / \mathrm{ml}$ for Asian and ESCA strain respectively. The combinational MIC of Doxycycline and Ribavirin on Dengue subtypes 1, 3, and $4 \mathrm{were} 7.8 \mu \mathrm{g} / \mathrm{ml}$, and Dengue 2 was $3.9 \mu \mathrm{g} / \mathrm{ml}$. All the statistical analyses were highly significant at $\mathrm{P}<0.0001$. Enthused by the results on Dengue and Chikungunya, the antiviral efficacy of these drugs to SARS-CoV-2 was studied by in-silico assay in comparison with Remdesivir, Hydroxychloroquine, Chloroquine, Azithromycin, and Favipiravir by their relative docking efficiency.

Conclusion: Doxycycline and Ribavirin are found to be potentially safe and efficacious for the treatment of Dengue/Chikungunya and the novel combination of them is slightly superior to its individual effect. Also, Doxycycline and Ribavirin are considered as the most potential drug for further evaluation in the treatment of COVID-19.
\end{abstract}

Keywords: SARS-CoV-2; Dengue; Chikungunya Virus; in-vitro Assay; in-silico Assay 
Doxycycline and Ribavirin as Antiviral and Novel Combinational Drug to Treat Dengue, Chikungunya and as the Most Potent Antivirals for COVID-19

\section{Introduction}

In the last two decades, the morbidity and mortality rates due to the re-emerging RNA viruses have increased and pose a highly significant threat to health of humanity. About 70 per cent of emerging infections are established to be zoonotic, and two-thirds have originated from wild animals. The increasing contact with animals and arthropod vectors of viral infections had increased human susceptibility to infections by novel pathogens. The absence of specific immunity in these populations due to the emergence of more virulent forms after a period of quiescence as evidenced by the recent COVID-19 pandemic and higher rates of mortality; debilitating and prolonged arthralgia caused by CHIKV virus since 2006 and ZIKA viral encephalopathy in Brazil in 2015. Among the emerging viral infections, arboviral infections (Chikungunya virus, Dengue virus) respiratory viral infections (Pandemic influenza H1N1, avian influenza (AI) infection H5N1) represent the major categories of viral infections in India and in specific, the pandemic of COVID 19 throughout the world. The recent novel infectious disease, severe acute respiratory infection spread had originated and detected in Wuhan, China in December 2019 which rapidly started spreading all over the world endangering millions of human lives. To update as latest as $16^{\text {th }}$ of Dec 2021, high risk global death toll has extended up to 5345391 with 272469550 confirmed cases from 223 affected countries as reported by WHO [1]. 2\% fatality rate [2]. Reports are in public domain on deficiency of vaccines to Delta as well as to omicron due to infective ability SARS-CoV-2 in vaccinated population. Recent clinical trial brought out waning of anti-bodies after 6 months indicating the need for revaccination for every six months. In addition, the immunogenic ability of the vaccine is variable among individuals as only $50-55 \%$ of the vaccinated develop adequate antibodies, supported by reported infection among vaccinated healthy persons. Merk's Molnupiravir, anti-COVID19 drug for emergency use reported only 30\% efficacy in clinical trial [3]. Hence the need for potential anti-COVID-19 drug for treating vaccinated but infected population as well as in children, pregnant woman, immune deficient and compromised persons.

The novel Severe Acute Respiratory Syndrome Coronavirus-2 (SARS-CoV-2) is a positive-strand RNA virus with crown like appearance [4]. It is a non-segmented RNA genome packed with four major structural proteins (Envelope-E, Spike-S, Membrane-M and Nucleocapsid-N) arranged in the order of $5^{\prime}$-leader-UTR-replicaseS-E-M-N-3'UTR-poly(A) tail genome organization [5,6]. The patients are treated symptomatically, and besides, the current management of COVID-19 include self-isolation, travel restrictions and supportive medical care with the excellent frontline worker. Though vaccination for emergency use has been gaining momentum, lack of efficacious and safe antivirals is a critical factor in control and prevention of mortality [7]. Yet the mortality rate and infection rate raise unabated, naming it as second cave in the post COVID vaccine period. Therefore, foremost priority is to identify most potential antivirals for these viruses that exert both antiviral effect and the antagonistic effect for the management of disease complications. Our first evaluation was on finding safe and efficacious antivirals for CHIKV and Dengue serotypes through in-silico evaluation followed by in-vitro confirmation assay of two drugs (Doxycycline and Ribavirin) as monotherapy and combinational therapy.

On observing the efficacy of Doxycycline and Ribavirin, we extended our research on the current novel SARS-COV-2 through insilico studies to investigate the efficacy which could help for the current critical situation. The tested antivirals used in this study are Ribavirin, Doxycycline, Azithromycin, Remdesivir, Hydroxychloroquine, Favipiravir and Chloroquine on SARS-CoV-2 through in-silico studies. Among this Ribavirin and Doxycycline were studied against Dengue and CHIKV by in-vitro analysis. Few studies were done to find the effect of Ribavirin against Coronavirus by in-vitro and in-silico analyses, but remarkably none of these studies has evaluated on the recent deadliest strain [8-10]. Apparently, Doxycycline and Azithromycin are newly evaluated drugs for this SARS-CoV-2 virus. Nevertheless, the Doxycycline and Azithromycin are antibiotics; many earlier studies have examined these for many viruses due to their effective antagonistic activity [11-13]. Taken together, our results will provide resilient evidence to go further on experimental and clinical investigations towards treating and decreasing the SARS-CoV-2 morbidity and mortality rate on observing the efficiency of anti- SARS-CoV-2 properties.

\section{Materials and Methods}

Docking analysis of doxycycline against dengue and chikungunya virus by In-silico evaluation

The pre-evaluation of antiviral efficiency of Doxycycline was tested and predicted by in-silico studies. The drug of interest, Doxycycline has been tested for docking efficiency of Chikungunya and Dengue viral strains namely 3TRK and 1R6A. The structure of Doxycycline and active site of Dengue and CHIKV were depicted in Figure $1 \mathrm{a}$ - c respectively. The molecular docking with the Doxycycline was performed using Auto-dock 4.2.(13) Both the target protein and the ligand molecule were prepared using Pymol and open Babel respectively. To improve the binding affinity, water molecules were removed before docking experiments. The grid box within 
Doxycycline and Ribavirin as Antiviral and Novel Combinational Drug to Treat Dengue, Chikungunya and as the Most Potent Antivirals for COVID-19

the dimension with $\mathrm{X}=-26.3, \mathrm{Y}=12.5, \mathrm{Z}=59$ using Auto-grid of $0.547 \AA$ Å spacing was used to cover the entire receptor molecule so that there is sufficient space for the ligand to easily move flexibly within the binding pocket. The docking complex was obtained after respective docking compound of interest and the lowest binding energy value was selected as docked complex from the conformational dock and saved in PDB format. All the docked pores were analyzed carefully using Pymol and interactions between the ligand, and the receptor were studied. The Hydrogen bonds interactions less than $3.5 \AA$ was considered along with other non-bonded interactions.

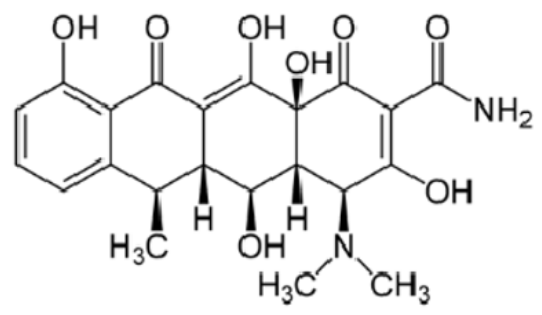

(a)

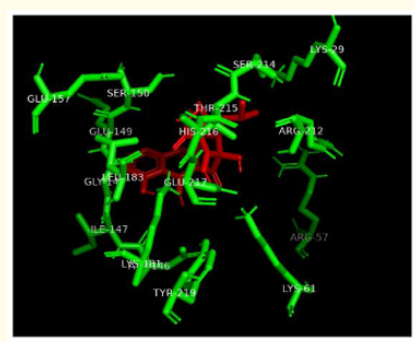

(b)

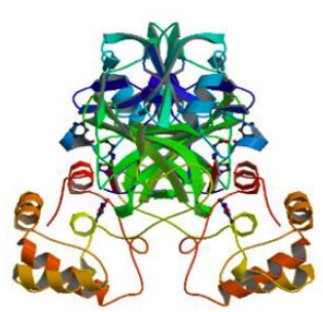

(d)

Figure 1a: Structure of Doxycycline.

Figure 1b: Active site of Dengue - PDB 1R6A.

Figure 1c: Active site of CHIKV- PDB 3TRK.

Figure 1d: Crystal structure of SARS-CoV-2 main protease - PDB 6 LU7.

Figure 1e: Active site of SARS-CoV-2.
Procurement of cells, viruses and compounds

Clinical isolates of Dengue serotypes (1-4) and CHIKV (Asian and ECSA strain) were procured from National Institute of Virology (NIV) Pune and used for viral propagation in Vero cell line which was procured from National center of cell Sciences (NCCS), Pune, India. Vero cell lines were cultured in Dulbecco's modified Eagle's medium (DMEM), supplemented with 10\% fetal bovine serum (FBS), (Sigma Aldrich) as a growth medium or $2 \%$ FBS as a maintenance medium with $100 \mathrm{mg} / \mathrm{ml}$ penicillin-G (Sigma), $60 \mathrm{mg} / \mathrm{ml}$ streptomycin (Sigma) and cultured for five days. Inoculated cells were incubated at $37^{\circ} \mathrm{C}$ at $5 \% \mathrm{CO}_{2}$ and split two times a week to maintain confluency. The cell debris of propagated standard strain of CHIKV and Dengue serotypes were removed by centrifugation at $3000 \mathrm{RPM}$ for 10 minutes, and the viral supernatant was aliquot in $2 \% \mathrm{FBS}$ and stored at $-80^{\circ} \mathrm{C}$. Ribavirin and Doxycycline were purchased from Sigma (Sigma Aldrich) and dissolved in DMSO to a final concentration of less than $1 \%$ of the total volume.

\section{Estimation of TCID50 to CHIKV and dengue serotypes}

About $100 \mu \mathrm{l}$ of the viral strain was suspended in $900 \mu \mathrm{l}$ of $2 \%$ MEM, and it was serially diluted from 1 to $12^{\text {th }}$ concentration, and $100 \mu l$ of the diluted virus was added to the confluent cell lines and incubated at $37^{\circ} \mathrm{C}$ for 1 hour in $5 \% \mathrm{CO}_{2} .100 \mu l$ of $2 \%$ minimum essential medium was added. After $48 \mathrm{hrs}$, the cytopathic effect (CPE) in the wells of each row was counted, and TCID 50 was estimated as below.

$\operatorname{TCID50}=$

log 10 dilution factor (\% infection at next dilution above 50\%-50)

(\% infection at next dilution 50\%-\% infection at Next dilution below $50 \%$ )

\section{Cell viability by in-vitro cytotoxicity assay}

After trypsinization, cells were dislodged with $10 \mathrm{ml}$ of $10 \%$ Minimum essential medium. About $100 \mu \mathrm{l}$ of the cells were added to the 96 well plates and was incubated at $37^{\circ} \mathrm{C}$ in $5 \% \mathrm{CO}_{2}$ for 24 hrs. After $24 \mathrm{hrs}$ incubation, the different working concentrations were added to each respective well and $100 \mu \mathrm{l}$ of $2 \%$ Minimum essential medium at $37^{\circ} \mathrm{C}$ in $5 \% \mathrm{CO}_{2}$ for $72 \mathrm{hrs}$. The present invention describes the combination of a semisynthetic antibiotic, Doxycycline and an antiviral agent Ribavirin in an equal proportion of 50:50 ratio and then preparing various dilutions of different concentrations. The initial individual drug concentration was from $500 \mu \mathrm{g} / \mathrm{ml}$ for Doxycycline and Ribavirin while the combined drug concentration was from the dilution 250:250 $\mu \mathrm{g} /$ $\mathrm{ml}$ and hence processed with two-fold dilution until 1.95:1.95 $\mu \mathrm{g} /$ 
Doxycycline and Ribavirin as Antiviral and Novel Combinational Drug to Treat Dengue, Chikungunya and as the Most Potent Antivirals for COVID-19

$\mathrm{ml}$ concentration. was attained and incubated till 72 hours for the observation of cell viability. Maximum non-toxic dose (MNTD) of the single and combinational drugs were estimated by observing the cells that were as equivalent to that of Vero control cells under the Phase contrast inverted microscope.

\section{In-vitro viral inhibition assay}

Cell viability or cytopathic effect evaluation is usually employed mainly for the initial evaluation to find out the efficacy of the drug in CHIKV or many other viruses [14]. The antiviral activity of Doxycycline and Ribavirin was evaluated as a single agent and as a combinational drug determination against CHIKV and Dengue serotypes on Vero cells. The Vero cell line was more permissive to infection than to any other cell lines and hence lower MOI (Multiplicity of Infection) was necessary to ensure viral replication kinetics to remain similar in cell lines [15]. The confluent monolayer of 96 well Tissue culture plate was infected by Asian strain and African strain separately at MOI of 1 PFU/cell for Vero cell line. Similarly, Vero cells grown in 96 well Tissue culture plate were infected by Dengue virus serotypes 1,2,3 and 4 separately at MOI of 1 and allowed to adsorb the virus for 1 hour at $37 \circ \mathrm{C}$ with $5 \% \mathrm{CO} 2$.

After 1 hour, the monolayers were washed with PBS after removing the inoculum to remove the unbound virus. $100 \mu \mathrm{l}$ of the serially diluted different combinational drug from the maximum non-toxic concentration were inoculated into the infected Vero cells in the respective wells. The Vero cell exclusive of viral inoculum was taken as a mock control. After $72 \mathrm{hrs}$., the concentration of the compound that reduced the cytopathic effect of the virus and cell layer appearance as similar to that of the control was determined as the minimum inhibitory concentration (MIC) of the drug. The significant cytotoxic or cytostatic effects of Doxycycline and Ribavirin were evaluated and compared to uninfected cells by microscopic observation for any changes regarding induced virus-cytopathic effects in each applied concentration. All assays were performed in triplicates. The monotherapy and combinational therapy effect were determined and recorded accordingly.

In-silico evaluation for the most potent antiviral drug to SARSCoV-2

In-silico drug evaluation is an excellent fast track tool to evaluate and identify the therapeutic ability of a drug on any viruses by its binding ability and hydrogen bonding stability with the targeted protein of the Virus. The nucleocapsid (N) protein of SARS-CoV-2 plays a vital role in the incorporation of genomic RNA into progeny viral particles.

\section{Identification of target and compound structure}

Target. Protein identification is the main criteria to be focused on identifying the appropriate drug candidate in the initial step for drug designing procedure. With the help of various bioinformatic tools, the excellent drug could be identified. The X-ray crystal structure of SARS-CoV-2 main protease (PDB ID-6LU7), figure 1d was obtained from the Protein Data Bank (www.rcsb.org), which is an ideal target to be docked by the compound selected for this study. The active site of the main protease was demonstrated in figure 1e. The selected protein was complexed with N3, the potential protein involved in the viral replication and transcription, thus resulting as an attractive drug target for SARS-CoV-2 strain (16-18). The structures of Doxycycline, Ribavirin, Remdesivir, Chloroquine, Hydroxychloroquine, Azithromycin and Favipiravir were retrieved from PubChem https://pubchem.ncbi.nlm.nih.gov/. The protocol and speculations of molecular docking with the compounds of interest were followed as mentioned in 1.2.1.

\section{Statistical analysis}

All the assays were done in triplicate, and the Mean and standard error of the mean (SEM) from three independent experiments were calculated. Statistical analyses were performed by using Graph-Pad Prism 6 Software (GraphPad Software, Inc.). A p-value $<0.05$ was considered statistically significant.

\section{Results and Discussion}

In-silico docking analysis of doxycycline against dengue and CHIKV virus

Molecular docking as a pre-laboratory antiviral efficacy was identified by virtual screening. The compound Doxycycline had high energy interaction towards both Dengue and Chikungunya with 7 and 8 hydrogen bond interactions with different amino acids with highest binding energy The Doxycycline had docked successfully on both Dengue and Chikungunya virus with the binding efficiency of $-7.9 \mathrm{kcal} / \mathrm{mol}$ and $5.58 \mathrm{kcal} / \mathrm{mol}$ respectively. The active site of Chikungunya had docked with 5 hydrogen bonds with the amino acids whereas the active site of Dengue virus has docked with amino acids Methionine and Aspartic acid whereas the active site of Dengue virus had docked with amino acids Fly-148, Lys-181 and Glu-217 which was depicted in figure $2 \mathrm{a}$ and $\mathrm{b}$. Based on these identifications, the antiviral efficacy of Doxycycline was confirmed by in-vitro investigation on comparing with a standard RNA antiviral agent, Ribavirin. 


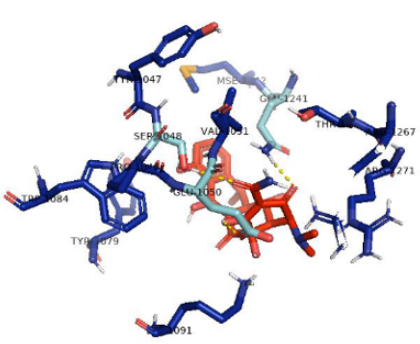

(a)

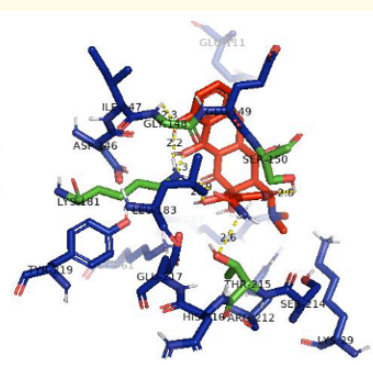

(b)
Figure 2a and 2b : Docking analysis of Doxycycline against CHIKV and Dengue virus.

The interaction between doxycycline against Chikungunya and

Dengue virus depicts the docking site with susceptible amino acids.

In-vitro antiviral activity of doxycycline and ribavirin to chikungunya virus

Doxycycline was non-toxic to Vero cells up to the concentration of $2000 \mu \mathrm{g} / \mathrm{ml}$ whereas Ribavirin was non-toxic to the cells up to $500 \mu \mathrm{g} / \mathrm{ml}$ and the combinational dosage of Doxycycline and Ribavirin were non-toxic up to the concentration of $1000 \mu \mathrm{g} / \mathrm{ml}$. Supplementary figure 1 represents the toxicity results (MNTD) for Doxycycline and Ribavirin. The antiviral activity of Doxycycline and Ribavirin was first evaluated as a single agent against Chikungunya virus and compared these with combinational therapy against CHIKV Asian and ECSA strains. The infected cells were exposed to compounds for four days in Vero cells and chose the day after peak viral burden as the endpoint of the assay. On observing the cytopathic changes, exciting results were observed, which are tabulated in table 1 and figure $3 \mathrm{a}$ and b. Doxycycline inhibited the Asian genotype of Chikungunya virus at a MIC of $15.62 \mu \mathrm{g} / \mathrm{ml}$ and Ribavirin at a MIC of $125 \mu \mathrm{g} / \mathrm{ml}$. The Minimum inhibitory concentration of Doxycycline on CHIKV ESCA was $62.5 \mu \mathrm{g} / \mathrm{ml}$, and disappointingly Ribavirin did not inhibit this strain. Though the combinational drug did not evince enhanced activity by synergistically inhibiting the Asian genotype a Chikungunya virus at a MIC of $31.5 \mu \mathrm{g} / \mathrm{ml}$, the combinational drug had the advantage of inhibiting ECSA genotype of Chikungunya despite the lack of inhibition of it by Ribavirin individually as demonstrated at $62.5 \mu \mathrm{g} / \mathrm{ml}$.

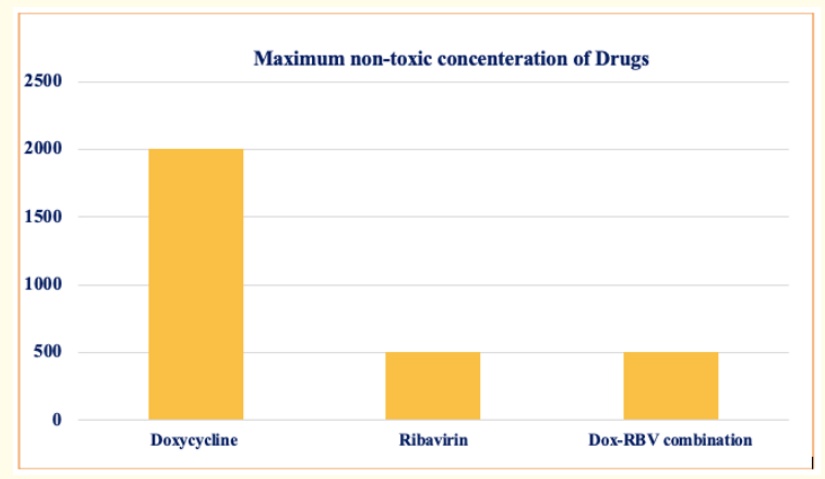

Supplementary Figure 1: Cytotoxicity assay for Doxycycline and Ribavirin.

The non-toxic concentration, $\mathrm{CC}_{50}$ of Doxycycline $(2000 \mu \mathrm{g} / \mathrm{ml})$, Ribavirin $(500 \mu \mathrm{g} / \mathrm{ml})$ and combinational Dox-RBV was 500 $\mu \mathrm{gg} / \mathrm{m}$.

\begin{tabular}{|l|c|c|c|}
\hline Virus & Doxycycline & Ribavirin & $\begin{array}{c}\text { Doxycycline and Ribavirin } \\
\text { combination }\end{array}$ \\
\hline Asian strain of CHIKV & 125 & 125 & $62.5: 62.5$ \\
\hline East Central South African strain of CHIKV & 62.5 & - & $125: 125$ \\
\hline Dengue virus type 1 & 62.5 & 15.6 & $7.8: 7.8$ \\
\hline Dengue virus type 2 & 62.5 & 7.8 & $3.9: 3.9$ \\
\hline Dengue virus type 3 & 125 & 15.6 & $7.8: 7.8$ \\
\hline Dengue virus type 4 & 62.5 & 15.6 & $7.8: 7.8$ \\
\hline
\end{tabular}

Table 1: Monotherapy inhibitory activity of Dox and RBV against CHIKV and Dengue serotypes. 

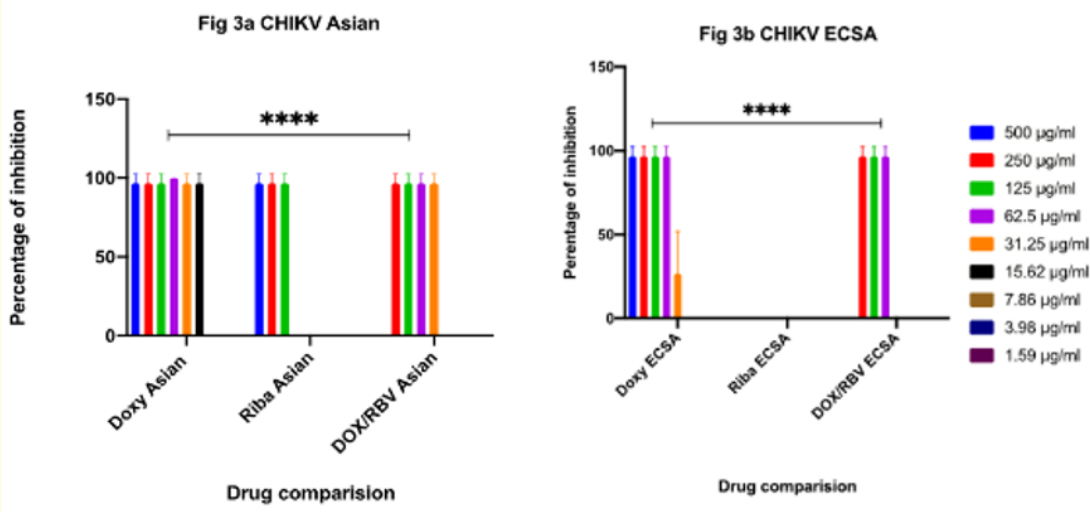

Figure 3a and 3b : Antiviral Inhibition assay on chikungunya virus.

The efficiency of Doxycycline, Ribavirin and combinational therapy on Chikungunya Asian strain: $p<0.0001^{* * * *}$ Figure $3 b$ : The efficien-

In-vitro antiviral activity of doxycycline and ribavirin to dengue virus

The superior efficiency of combinational drug modulation over monotherapy against Dengue serotypes were depicted in figure 4 $\mathrm{a}, \mathrm{b}, \mathrm{c}$ and $\mathrm{d}$ and table 1. The MIC of Doxycycline was at $62.5 \mu \mathrm{g} / \mathrm{ml}$ on Dengue 1, 2 and 4 serotypes and $125 \mu \mathrm{g} / \mathrm{ml}$ on Dengue 3. Similarly, the MIC of Ribavirin was at the concentration of $15.62 \mu \mathrm{g} / \mathrm{ml}$ against Dengue 1, 2 and 4 while Dengue 3 serotype was susceptible at the MIC of $31.25 \mu \mathrm{g} / \mathrm{ml}$. The Ribavirin has exhibited slightly superior antiviral activity to all Dengue serotypes when compared to the Chikungunya virus by its' lower MIC value. The combinational drug had shown enhanced activity by synergistically inhibiting the Dengue 1, 2 and 4 at the MIC of $7.86 \mu \mathrm{g} / \mathrm{ml}$ while Dengue 3 was inhibited synergistically at a lower MIC of $3.98 \mu \mathrm{g} / \mathrm{ml}$. All result data were statistically compared for significance. It was highly significant between monotherapy and combinational therapy against all Dengue viral serotypes $\mathrm{p}<0.0001^{* * * *}$. The overall comparative efficacy of test drugs is depicted as a line graph (Supplementary Figure 2 ). The sample of illustration of drug-resistant and drug-sensitive cell line against Chikungunya and Dengue viral serotypes has been demonstrated in figure 5 . The partial inhibition of respective viral strains also has been shown in histogram charts.

\section{Molecular docking results on SARS-CoV-2}

Lipinski's rule of 5

The rule of 5(R05), a rule of thumb will be used to evaluate the drug-likeness of any compound of interest. We have analyzed all our compound of interest to evaluate the R05 to adopt the rules that are, hydrogen-bond-donating atoms $>5$, the ligand with $\mathrm{MW}>500 \mathrm{~g} / \mathrm{mol}$, hydrogen-bond-accepting atoms $>10$, or Clog $\mathrm{P}>5$. The violation of the Lipinski rules was neglected as the drug of interest is already widely used for treatment regime for many diseases, Therefore, all drugs were taken for in-silico antiviral analysis on SARS-CoV-2 main protease despite their few violations to the Lipinski rules.

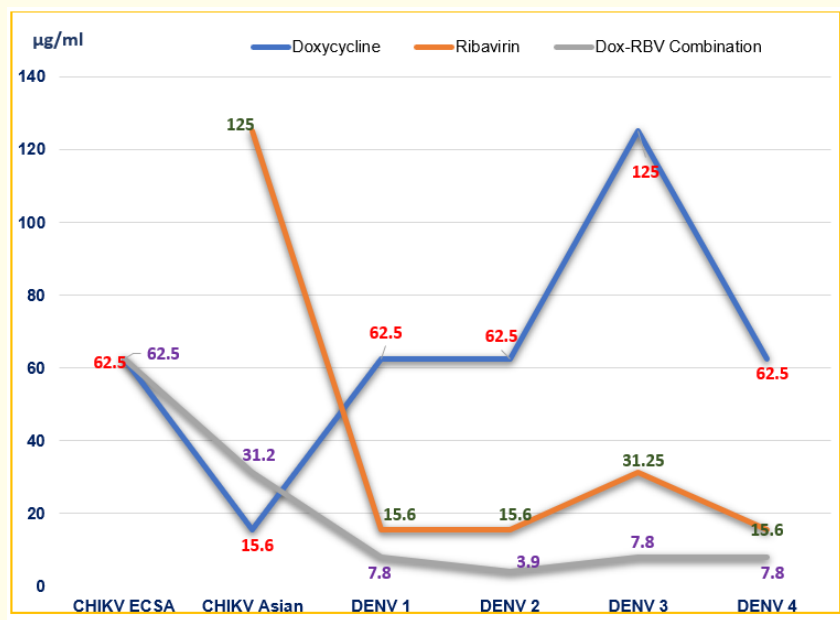

Supplementary Figure 2 : Line graph on MIC concentration of Doxycycline, Ribavirin and Combination therapy for all strains.

Line graph explains the inhibitory activity of Doxycycline and Ribavirin monotherapy and Combinational therapy with the appropriate effective concentration. 

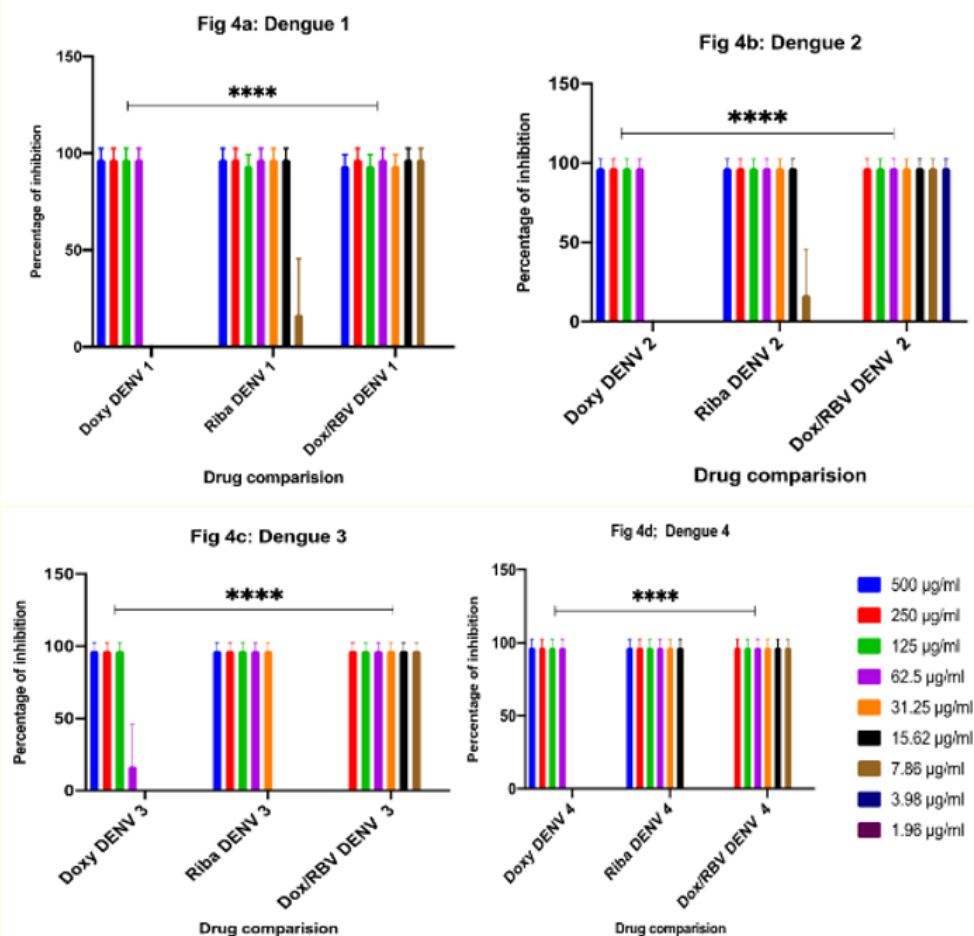

Figure 4a, b, $\mathbf{c}$ and d: Antiviral Inhibition assay on Dengue virus.

Figure 4a: The efficiency of Doxycycline, Ribavirin and Combinational therapy on Dengue serotype $1 \mathrm{p}<0.0001^{* * * *}$ Figure $4 \mathrm{~b}$ : The efficiency of Doxycycline, Ribavirin and Combinational therapy on Dengue serotype $2 \mathrm{p}<0.0001^{* * * *}$ The efficiency of Doxycycline, Ribavirin and Combinational therapy on Dengue serotype $3 p<0.0001^{* * * *}$ The efficiency of Doxycycline, Ribavirin and Combinational therapy against Dengue serotype $4 \mathrm{p}<0.0001^{* * * *}$ Data from triplicate assays were plotted using Two-way annova, Graph Pad Prism Version 5 (Graph Pad Software Inc., San Diego, CA. USA).

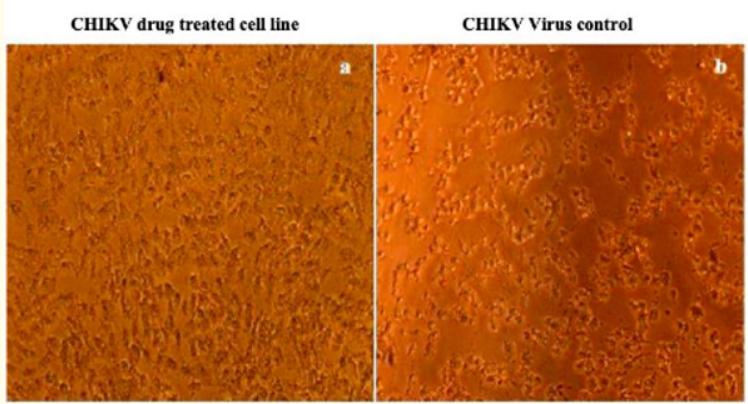

Dengue virus control

Drug treated cell line

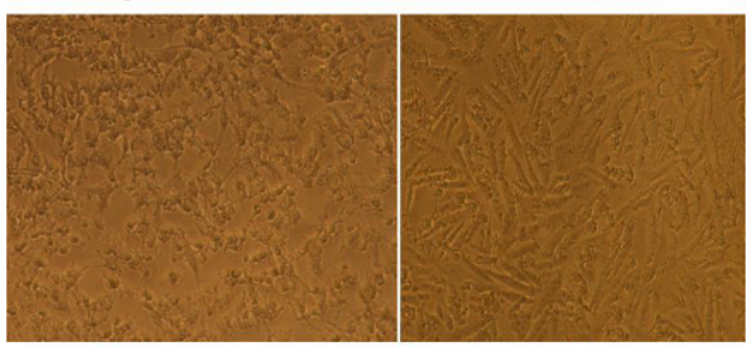

Figure 5: In-vitro antiviral efficacy of Doxycycline and Ribavirin on Chikungunya and Dengue virus.

Illustration of drug treated cell line and drug-free cell line for Dengue and Chikungunya virus. The drug-free cell line picture has depicted as virus control of respective virus. 
Doxycycline and Ribavirin as Antiviral and Novel Combinational Drug to Treat Dengue, Chikungunya and as the Most Potent Antivirals for COVID-19

Docking score and hydrogen bond interaction

The docking profile of all the compounds and binding efficiency against SARS-CoV-2 was represented in figure 6(a-g) and table 2. In China, the compound Remdesivir was tried as a treatment to fight novel coronavirus (SARS-CoV-2). The compound Remdesivir despite exhibiting high interaction energy it has only one hydrogen bonding. Similarly, Favipiravir has also exhibited nearest binding energy to Remdesivir as $-5.5 \mathrm{kcal} / \mathrm{mol}$ with the active sites THR292, ASN151, THR111, ASP295 with 5 hydrogen bonds. However, Doxycycline not only exhibited slightly higher binding energy but had multiple hydrogen bonding reflecting strong stability. Ribavirin has showed the highest binding energy as well as the highest number of hydrogen bonding compared to other compounds. The intensity of docking and binding energy were studied using Auto-dock 4, and the results are tabulated in table 2 . Among all the tested antivirals, Ribavirin showed the highest binding energy of $-6.4 \mathrm{kcal} / \mathrm{mol}$ with seven hydrogen bond interactions. Similarly, the drug doxycycline exhibited binding energy of $-5.7 \mathrm{kcal} / \mathrm{mol}$ with five hydrogen bonds. A comparative graphical representation of binding energy and hydrogen bond stability, exhibited by test drugs, in docking against SARS-CoV-2 is shown in figure 7.

\begin{tabular}{|c|c|c|c|c|}
\hline S. No & Ligands & $\begin{array}{c}\text { Binding Energy } \\
\text { Kcal/mol }\end{array}$ & Hydrogen bond interactions & $\begin{array}{c}\text { No of HB } \\
\text { bonds }\end{array}$ \\
\hline 1 & Remdesivir & -5.6 & LYS137 & 1 \\
\hline 2 & Doxycycline & -5.7 & LYS5 & 5 \\
\hline 3 & Ribavirin & -6.4 & ASP295, ASN151, SER158, GLN110, & 7 \\
\hline 4 & Chloroquine & -4.9 & THR111, LYS102 & 2 \\
\hline 5 & Hydroxychloroquine & -5.5 & GlN110, THR111, SER158 & 3 \\
\hline 6 & Azythromycin & -5.5 & LYS5 & 2 \\
\hline
\end{tabular}

Table 2: Autodocking of drug of interest towards SARS-CoV-2.
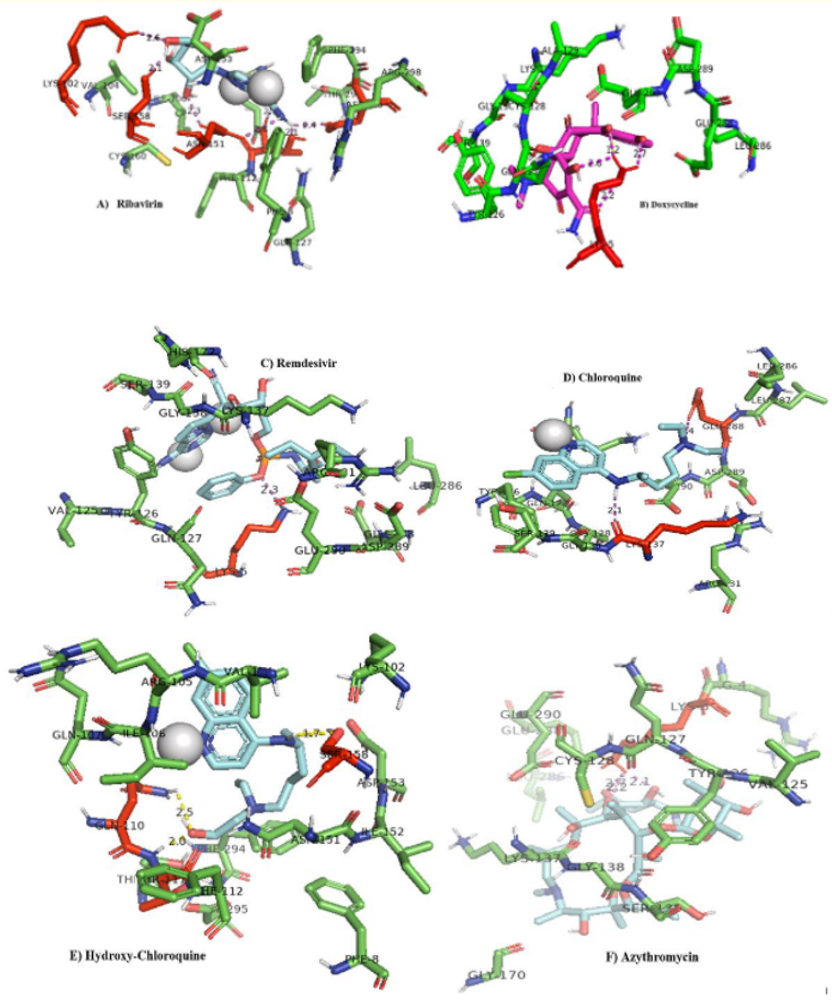

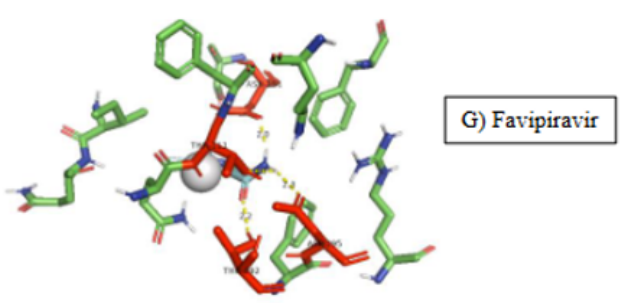

Figure 6 : Virtual screening and docking of selected compounds (A-F) against SARS-CoV-2 main protease PDB 6LU7.

A) The docking site of Ribavirin with amino acids ASP295, ASN151, SER158, GLN110, THR111, LYS102 B) The docking site of doxycycline with amino acid LYS5 C) The docking site of Remdesivir with amino acids LYS137 D) The docking site of Chloroquine with amino acids GLY138, ASP279, GLU288 E) The docking site of Hydroxychloroquine with amino acids GIN110, THR111, SER158

F) The docking site of Azythromycin with amino acid LYS5. 
Doxycycline and Ribavirin as Antiviral and Novel Combinational Drug to Treat Dengue, Chikungunya and as the Most Potent Antivirals for COVID-19

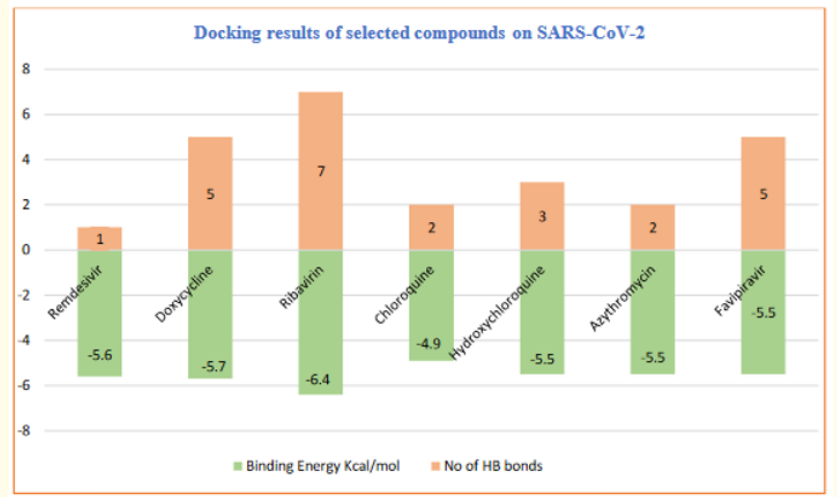

Figure 7 : In silico evaluation of docking score of selected drugs on SARS-CoV-2.

SARS-CoV-2, Dengue and Chikungunya are RNA viruses of serious disease-causing agents that result in morbidity or mortality in high-risk patients that deserve and demands rapid designing and discovery of novel drug/s for effective treatment. The lack of antiviral drugs and vaccines make it a formidable task in the control of this global epidemic (COVID19) and of the regular annual outbreaks of Chikungunya and Dengue, as current treatment strategies rely on the alleviation of disease symptoms. Therefore, in this study, we have evaluated a comprehensive antiviral activity of a few potential drugs on these viruses. The test drugs of interest in this study were Doxycycline, Ribavirin for Chikungunya and Doxycycline and test drugs for COVID19 were Doxycycline, Ribavirin, Remdesivir, Azithromycin, Hydroxychloroquine and Chloroquine. Among these drugs, Doxycycline, Azithromycin and Chloroquine are generally an antibiotic and antiparasitic drugs, but many research crews have published with promising results on the efficacy and binding energy of these towards these viruses [14].

Doxycycline and Ribavirin [15] have been known well for the antibiotic and non-antibiotic properties since its discovery. However, we have found that the novel combination of Doxycycline and Ribavirin in various dosages exhibited synergistic antiviral effect more particularly against all the serotypes of Dengue virus and appreciable inhibitory activity on Chikungunya both Asian and ECSA genotypes which is an outstanding finding of this study while there is no approved drug for the treatment of the two diseases till date. Also, these promising results provided a hint to our research team to examine their potency on SARS-CoV-2 strain as a fast-track evaluation by in-silico studies in comparison to few currently focused drugs for COVID-19. As the synergistic combinational evaluation on any virus through in-silico model is not possible, it may be verified by in-vitro analysis. For the comparative analysis, five more drugs of current relevance to COVID19 namely, Remdesivir, Favipiravir, Hydroxychloroquine, Chloroquine and Azithromycin were studied to know their binding energy against the main protease of SARSCoV-2 from PDB, which were deposited by the recent research crew [16].

In this study, we have analysed the efficacy of Doxycycline and Ribavirin on Chikungunya and Dengue serotypes as both monotherapies and in 1:1 combinational therapy starting from the concentration of $500 \mu \mathrm{g} / \mathrm{ml}$. The complete minimal inhibitory concentration of synergistic combination activity of Dengue and Chikungunya was appreciable. Among these Doxycycline has exhibited superior inhibitory activity over Ribavirin on both strains of CHIKV while Ribavirin has exhibited slightly superior inhibitory activity over Doxycycline on all four subtypes of Dengue. Combinational drug of Doxycycline and Ribavirin in equal proportion has the advantage of treating both genomic types of Chikungunya due to lack of antiviral activity by Ribavirin on ECSA CHIKV. Rothan., et al. had studied the antiviral effect on ESCA strain alone while this study has evaluated the antiviral efficacy on both ECSA and Asian strain [17].

Rothan., et al. 2013 had also studied the monotherapy antiviral activity on Doxycycline and Ribavirin separately against all four serotypes of Dengue virus and reported that Doxycycline in monotherapy post-incubation evaluation, has showed better activity on DENV 2 and 4 than DENV 1 and 3 [12]. However, the present study had revealed appreciable inhibitory activity of the novel combination Doxycycline and Ribavirin on all serotypes of Dengue virus. This is a novel finding and core strength of this research work. Ribavirin is a prevailing antiviral agent which is actively inhibiting many viruses has shown its appreciable activity especially on all the three viruses included in this study. RBV had the most significant antiviral effect EC50 at $2.575 \mathrm{mg} / \mathrm{mL}$ in Huh-7 cells [18] while the current study has analyzed its efficacy on Vero cell line. It has shown the excellent inhibitory activity against all dengue viral strains and CHIKV Asian strain except ECSA strain. Additionally, it has shown extremely inhibitory activity against SARS-CoV-2 main protease with the best binding energy among all other compounds through in-silico analysis (Auto docking) analysis.

The in-vivo safety combination of Doxycycline and Ribavirin (1:1) has been studied in mice for its acute toxicity, and the results indicated its safety. In addition, combinational in-vivo antiviral efficacy of Doxycycline and Ribavirin on CHIKV ECSA strain alone 
Doxycycline and Ribavirin as Antiviral and Novel Combinational Drug to Treat Dengue, Chikungunya and as the Most Potent Antivirals for COVID-19

is compared with monotherapy groups (Ribavirin) [17]. The viral infection seemed to have only partially cleared in monotherapy group indicated by a partial reduction in abnormal enlargement in size and increase in weight of liver and spleen while combinational drug resulted in near-complete clearance of virus due to restoration of normal weight and size of liver and spleen [17]. It is clear from this study, that though Ribavirin has lower MIC values than Doxycycline on Dengue virus, the MIC of the combinational drug was much lower than that of Ribavirin alone indicating a synergistic effect. This could be partially explained by the observation of Rothan., et al. through in-vivo analysis in mice for CHIKV ECSA that the inhibitory activity of Doxycycline is mainly at entry-level and activity at the Ribavirin is at the post entry-level, namely inhibition of RNA replication process. In addition, it has been observed that uptake of Ribavirin is lowered or hampered due to autophagic changes in the liver due to viral infection and the correct combinational drug showed better efficacy [17].

Also, the anti-inflammatory features of Doxycycline are an added advantage in relieving the inflammatory changes in the liver and spleen [19]. Finally, the cost of the drug as a combinational drug will be feasible due to the affordable cost of Doxycycline. As reasonable safety of the combinational drug has been ensured in acute toxicity study conducted in mice for CHIKV ECSA strain and as no animal model is available for in-vivo safety study for Dengue, clinical trial on this combinational drug is strongly suggested to confirm its therapeutic efficacy and safety in human. Hence it is highly deducible that Doxycycline and Ribavirin as the combinational drug is the most potential candidate drug to treat all strains of Chikungunya and all serotypes of Dengue infection.

Consequently, we performed an in-silico analysis for the compounds Ribavirin, Doxycycline and parallelly checked for the compounds Azithromycin as it is an analogue of erythromycin. Overall, we have studied six different compounds against SARS-CoV-2 using Auto dock. Few exciting features were found in the interaction profile of different compounds (i.e.) Doxycycline and Azithromycin formed multiple hydrogen bonds with the amino acid LYS5. The pattern of interaction was also the same, and the compound Hydroxychloroquine also found to interact with LYS5. The interaction profile of Hydroxychloroquine and Ribavirin showed a similar interaction profile forming hydrogen bonding with SER158, GLN110, THR111 but Ribavirin showed the effective interaction with other amino acids like ASP295, ASN151 and LYS102. A similar in-silico study was performed by Kandeel., et al. on the main protease has recommended Ribavirin, telbivudine, vitamin B12 and nicotinamide as potential compounds and reported that GLN 189, THR 25 were actively involved in hydrogen bonding followed by GLN 189 for telbivudine [20]. Ribavirin, Remdesivir, Sofosbuvir, Galidesivir, and Tenofovir have been studied for their efficiency on docking module in binding with RdRp protein of SARS-CoV-2 [21]. Therefore, based on these in-silico, in-vitro evaluations, this study strongly recommends Ribavirin and Doxycycline as promising antiviral candidate drugs to treat all these RNA Viral diseases based on the in-silico assays. Hence, to bring out the potential drug for COVID19, the in-vitro anti-SARS-Cov-2 activity of Ribavirin and Doxycycline must be performed to enable pre-clinical trials to fast track in finding a promising and safe drug to effectively treat and control COVID19.

The pandemic has been a year long, SARS-CoV-2 has expressed susceptibility to many test drugs either in in-silico, or in-vitro and case studies $[22,23]$. The efficacy of prophylactic and therapeutic Remdesivir treatment against SARS-CoV-2 has worked with 24 hour post-incubation on reducing the clinical signs and replication in the lungs with a decreased severity of lung lesions [23]. This study has also analyzed the efficacy of Remdesivir in SARS-CoV-2 main protease through molecular docking, in which the binding energy has exhibited as $-5.6 \mathrm{kcal} / \mathrm{mol}$ with one LYS137 hydrogen bond interaction. Since the authors have specified that the Remdesivir has worked after 12-hour post-inoculation intensely, it could suggest that the mode of action of Remdesivir might be on nucleocapsid protein. This is emphasized because the Remdesivir has worked with our protein of interest, which is a nucleocapsid protein, main protease (genomic material). However, its' binding energy level and only one hydrogen bonding stability is substantially weaker than that of the Ribavirin and Doxycycline. This may be attributed to the unsatisfactory efficacy of Remdesivir in the recently concluded clinical trial conducted in U.S.A. Though, Remdesivir and Favipiravir had been approved for emergency used Drug regulatory authorities, WHO, FDA, ICMR are now skeptical of its use in the prevention of mortality and felt it's utility in just slightly reducing the recovery period. However more potential drugs like Ribavirin and Doxycycline for COVID-19 are lagging for want of experimental and clinical trial. Favipiravir showed better therapeutic responses (Day 1: $1600 \mathrm{mg}$ twice daily; Days 2-14: $600 \mathrm{mg}$ twice daily) than LPV/RTV on SARS-CoV-2 in terms of disease progression and viral clearance [24]. The docking score of Favipiravir in this study has been slightly lower than Remdesivir at $-5.5 \mathrm{kcal} / \mathrm{mol}$ at the active amino acid sites THR292, ASN151, THR111, ASP295 which is an appreciable result. The study has preferred Remdesivir and Favipiravir over chloroquine and Ribavirin based on their higher selec- 
Doxycycline and Ribavirin as Antiviral and Novel Combinational Drug to Treat Dengue, Chikungunya and as the Most Potent Antivirals for COVID-19

tivity index over chloroquine and Ribavirin [25]. Incidentally the study has reported in-vitro anti SARS-CoV-2 efficacy of Ribavirin at $(109.5 \mu \mathrm{M})$. However, the study didn't consider about the weaker binding ability of Remdesivir to SARS-CoV-2 with only one Hydrogen bonding while Ribavirin has binding ability of $-6.4 \mathrm{kcal} / \mathrm{mol}$ with 7 hydrogen bond interaction. Favipiravir also has lesser binding ability and lesser hydrogen bond interaction than Ribavirin (Table 2). In-silico anti-SARS-CoV-2 binding ability of Doxycycline is again much superior to Remdesivir and slightly superior to Favipiravir (Figure 6g). However in-vitro anti-SARS-CoV-2 experimental confirmation is the need of the hour.

The usefulness of hydroxychloroquine in either prevention or treatment of COVID19 is considered risky in the absence of medical supervision due to its severe side effects namely elevation in heart rate, renal failure and heart attack during the pre-clinical trials in USA which is declared from FDA but informed to provide $800 \mathrm{mg}$ on first day and followingly slow down the dose and particularly it could be administered above $50 \mathrm{~kg}$ or more weighed patients [26]. Yet it's use had been discontinued by WHO, FDA, ICMR. Hence further studies on Doxycycline are strongly suggested on in-vitro level against SARS-CoV-2 activity followed by a pre-clinical study before subjected to clinical trial. As the routine development of the safe and efficacy vaccine was expected to take many months for human use in all age groups. Drug regulatory authorities had approved use of few vaccines for emergency use only despite partial completion of clinical trial. Apparently, these vaccines have only short duration of few months' efficacy and few adverse effects such as mortality due to blood clot. The post COVID-19 vaccine surge in infection and mortality mounts to a historical high to call it as second wave of COVID-19, immediate intervention in identifying appropriate antivirals is of utmost concern.

Interestingly, increase in the level of Interleukin- 6 has been reported as a biomarker for progression of disease in COVID19 patients. Abnormal increase in IL-6 also indicates necessity of ventilator facility [27]. Impact of Ribavirin has been studied on viral reduction in SARS-CoV-1 in correlation with IL-6 and Necrosis Factor [28]. It was found that indifferent to reduction of viral load, Ribavirin was not found to be efficient in reduction of IL-6 [28]. However, doxycycline and Levofloxacin individually was found to be effective in control of abnormal increase of IL- 6 in Dengue patients and human sinovocytes infected cultures with Chlamydia trachomatis respectively [29,30]. These bring out that Doxycycline would be preferable to Ribavirin in the COVID19 treatment due to its dual role in the reduction of viral load and also in the control of elevation of IL- 6 .

\section{Strength and limitations of this study}

The primary strength of this study was the establishment of the valuable use of in-silico screening Ribavirin and Doxycycline in predicting their antiviral efficacy to Dengue, Chikungunya viruses followed by convincing confirmatory observations by in-vitro assay. Perhaps this is the first in-silico -cum- in-vitro study combining dry lab and wet lab assays. Secondly, novel finding of combinational efficacy of drug Ribavirin and Doxycycline towards RNA viruses (CHIKV and Dengue serotypes) especially of the significant synergistic effect on all four subtypes of Dengue. The first in-silico, docking study of Doxycycline against Dengue and CHIKV virus. In addition, the first in-vitro, combinational antiviral study on all Dengue serotypes and both strains of CHIKV while there is no approved drug for the treatment of Dengue and Chikungunya. Equally unique is our intuition to test their (Doxycycline and Ribavirin) anti-SARS-CoV-2 potential by their docking affinity through in-silico assay and the desirable outcome over controls while none of the research crews has worked on it and compared these with other standard drugs such as Remdesivir, Azithromycin, Chloroquine, Favipiravir and Hydroxychloroquine. The final strength is the justification through statistical interpretation that all the interpretations have been found to be statistically highly significant. The main limitations of this study were non-availability of SARS-CoV-2 clinical strain to perform the in-vitro studies. We hope to evaluate in-vitro investigation and publish it sooner.

\section{Conclusion}

In the view of alarming infectivity and death rate of COVID-19 even after the approved premature use of vaccination for emergency use by regulatory authorities, reports of short duration efficacy, rare but fatal blood clotting side effects potential antivirals, Ribavirin and Doxycycline provide immediate solution awaiting speedy clinical trial on the therapeutic efficacy individually and in the combinational drugs to treat COVID-19. Among these two drugs, Doxycycline is suggested as the most potential candidate to treat COVID-19 due to its antiviral activity and efficacy to control IL-6 elevation. Collectively, the findings justify the use in-silico screening in identifying the antiviral efficacy of these drug candidates for treatment of Covid19, Dengue and Chikungunya. Also, this study underlines the significant synergistic efficacy of the combinational drug (Doxycycline and Ribavirin) especially against all Dengue serotypes and of appreciable inhibitory activity on both genotypes of CHIKV and possible potent antiviral efficacy on SARS-CoV-2. Doxycycline and Ribavirin have individually exhibited intense binding energy with a higher level of hydrogen bonding stability 
Doxycycline and Ribavirin as Antiviral and Novel Combinational Drug to Treat Dengue, Chikungunya and as the Most Potent Antivirals for COVID-19

to dock against the main protease of SARS-CoV-2. As synergistic effect could not be determined through in-silico studies, both these drugs are highly recommended for evaluation of their efficacy as monotherapy and combinational therapy against SARS-CoV-2 by in-vitro and in-vivo studies. Owing to the in-silico binding superiority of these two drugs in clinical use over the pre-clinical trial drugs for COVID19 namely Remdesivir, Hydroxychloroquine, Favipiravir and Azithromycin, experimental and pre-clinical studies of these compounds are to be conducted for WHO, FDA, ICMR approval for their clinical use in the successful treatment of the patients. The practice of highly efficacious combinational drug Doxycycline and Ribavirin is suggested for the treatment of Dengue and Chikungunya after clinical trial to ensure combinational safety and efficacy of this drug in comparison with single-drug group Doxycycline, Ribavirin for which safety dosage in human is known.

\section{Acknowledgement}

We acknowledge PG and Research Department of Microbiology, Presidency College (Aut.), University of Madras, Chennai, India for providing basic facilities with laboratory setup.

\section{Funding}

We would like to acknowledge the support of the Bioinformatics Division of Department of Biotechnology, Govt of India, for funding this project and utilisation of Bioinformatic Infrastructure facility Centre and Department of Science and Technology, Govt of India for the support on infrastructure and laboratory equipment on antiviral assay.

\section{Conflict of Interest}

The author has no conflict of interest.

\section{Author's Contribution}

RS conceived the research objective and guided the research crew. SV and SS conducted the in-vitro study and provided the inputs on writing the manuscript and IP conducted in-silico analysis and participated in drafting the manuscript. RS monitored the laboratory and in-silico analysis and approved based on the significant findings. All authors have reviewed the whole manuscript and provided equal responsibility.

\section{Bibliography}

1. WHO: Coronavirus death report - WHO. In., 25-4-2020 edn (2020).

2. Xu Z., et al. "Pathological findings of COVID-19 associated with acute respiratory distress syndrome". Lancet Respiratory Medicine 8.4 (2020): 420-422.
3. Kozlov M. "Merck's COVID pill loses its lustre: what that means for the pandemic". In: Nature 13 (2021).

4. Maheswari S., et al. "Air pollution enhances susceptibility to novel coronavirus (COVID-19) infection - an impact study". Environmental Analysis Health and Toxicology 35.4 (2020): e2020020-2020020.

5. Haagmans BL and Osterhaus AD. "Coronaviruses and their therapy”. Antiviral Research 71.2-3 (2006): 397-403.

6. Fehr AR and Perlman S. "Coronaviruses: an overview of their replication and pathogenesis". Methods in Molecular Biology 1282 (2015): 1-23.

7. Sabarimurugan S., et al. "Comprehensive review on the prevailing COVID-19 therapeutics and the potential of repurposing SARS-CoV-1 candidate drugs to target SARS-CoV-2 as a fast-track treatment and prevention option". Annals of Translational Medicine's 8.19 (2020): 1247.

8. Stroher U., et al. "Severe acute respiratory syndrome-related coronavirus is inhibited by interferon- alpha". Journal of Infectious Diseases 189.7 (2014): 1164-1167.

9. Morgenstern B., et al. "Ribavirin and interferon-beta synergistically inhibit SARS-associated coronavirus replication in animal and human cell lines". Biochemical and Biophysical Research Communications 326.4 (2005): 905-908.

10. Ferron F, et al. "Structural and molecular basis of mismatch correction and ribavirin excision from coronavirus RNA". Proceedings of the National Academy of Sciences of the United States of America 115.2 (2018): E162.

11. Castro JE., et al. "Modulation of cytokine and cytokine receptor/antagonist by treatment with doxycycline and tetracycline in patients with dengue fever". Clinical and Developmental Immunology 2011 (2011): 370872.

12. Rothan HA., et al. "Inhibitory effect of doxycycline against dengue virus replication in vitro". Archives of Virology 159.4 (2014): 711-718.

13. Menzel M., et al. "Azithromycin induces anti-viral effects in cultured bronchial epithelial cells from COPD patients". Scientific Report 6 (2016): 28698-28698.

14. Colson P., et al. "Chloroquine for the 2019 novel coronavirus SARS-CoV-2". International Journal of Antimicrobial Agents 55.3 (2020): 105923.

15. Sabarimurugan S., et al. "Computational screening of dual inhibitors from FDA approved antiviral drugs on SARS-CoV-2 spike protein and the main protease using molecular docking approach". Acta Virology 65.2 (2021): 160-172. 
16. Zhang L., et al. "Alpha-Ketoamides as Broad-Spectrum Inhibitors of Coronavirus and Enterovirus Replication: StructureBased Design, Synthesis, and Activity Assessment". Journal of Medicinal Chemistry (2020).

17. Rothan HA., et al. "A combination of doxycycline and ribavirin alleviated chikungunya infection". PLoS One 10.5 (2015): e0126360.

18. Franco EJ., et al. "The effectiveness of antiviral agents with broad-spectrum activity against chikungunya virus varies between host cell lines". Antiviral Chemistry and Chemotherapy 26 (2018): 2040206618807580.

19. Di Caprio R., et al. "Anti-Inflammatory Properties of Low and High Doxycycline Doses: An <i>In Vitro</i> Study". Mediators of Inflammation 2015 (2015): 329418.

20. Kandeel M and Al-Nazawi M. "Virtual screening and repurposing of FDA approved drugs against COVID-19 main protease". Life Science 251 (2020): 117627.

21. Elfiky AA. "Ribavirin, Remdesivir, Sofosbuvir, Galidesivir, and Tenofovir against SARS-CoV-2 RNA dependent RNA polymerase (RdRp): A molecular docking study". Life Science (2020): 117592.

22. Tian X., et al. "Potent binding of 2019 novel coronavirus spike protein by a SARS coronavirus-specific human monoclonal antibody". Emerging Microbes and Infections 9.1 (2020): 382385 .

23. de Wit E., et al. "Prophylactic and therapeutic remdesivir (GS5734) treatment in the rhesus macaque model of MERS-CoV infection". Proceedings of the National Academy of Sciences of the United States of America 117.12 (2020): 6771.

24. Cai Q., et al. "Experimental treatment with favipiravir for COVID-19: an open-label control study". Engineering (2020).

25. Wang M., et al. "Remdesivir and chloroquine effectively inhibit the recently emerged novel coronavirus (2019-nCoV) in vitro". Cell Research 30.3 (2020): 269-271.

26. FDA: Hydroxychloroquine (2020).

27. Ulhaq ZS and Soraya GV. "Interleukin- 6 as a potential biomarker of COVID-19 progression". Medecine et Maladies Infectiousness 50.4 (2020): 382-383.

28. Wang W-K., et al. "Temporal relationship of viral load, ribavirin, interleukin (IL)-6, IL-8, and clinical progression in patients with severe acute respiratory syndrome". Clinical Infectious Diseases : An Official Publication of the Infectious Diseases Society of America 39.7 (2004): 1071-1075.
29. Ikeda-Dantsuji Y., et al. "Effects of levofloxacin and doxycycline on interleukin- 6 production of Chlamydia trachomatis-infected human synovial fibroblasts". Chemotherapy 53.5 (2007): 332-337.

30. M Fredeking T., et al. "Dengue patients treated with doxycycline showed lower mortality associated to a reduction in IL-6 and TNF levels". Recent Patents on Anti-infective Drug Discovery 10.1 (2015): 51-58.

\section{Assets from publication with us}

- Prompt Acknowledgement after receiving the article

- Thorough Double blinded peer review

- Rapid Publication

- Issue of Publication Certificate

- High visibility of your Published work

Website: www.actascientific.com/

Submit Article: www.actascientific.com/submission.php

Email us: editor@actascientific.com

Contact us: +919182824667 